Ann. Biol. anim. Bioch. Biophys., 1977, 17 (4), 509-514.

\title{
Ammoniogenèse et motricité du rumen chez le mouton
}

\author{
par L. BUENO *, V. DOULOU *, M. CANDAU ** \\ * Laboratoire de Physiologie, Ecole Nationale Vétérinaire \\ 31076 Toulouse Cedex, France \\ ** Laboratoire de Zootechnie, Ecole Nationale Supérieure \\ Agronomique, 31000 Toulouse, France.
}

Summary. Effects of ammonia on rumen motility in sheep.

The effects of ammonia ( 400 mmoles, $\mathrm{pH} 8.8$ and 11.1), given by intraruminal and intravenous pathways, were studied in 4 fistulated sheep. Ammonia at $\mathrm{pH} 8.8$ and 11.1 when introduced into the rumen inhibited rumino-reticular contractions ; this effect, when compared with $\mathrm{NaOH}$ administration, was largely independent from the $\mathrm{pH}$ values. Hyperammonia, induced by infusion into the jugular or portal vein, had no effect. Specific local sensitivity of the rumen wall to free or ionized ammonia is discussed.

\section{Introduction.}

II est actuellement reconnu que le facteur majeur limitant l'efficience de l'utilisation de l'urée par les ruminants est la vitesse d'uréolyse dans le rumen, responsable de l'accumulation d'ammoniac et de l'élévation du $\mathrm{pH}$ dans le rumen (Repp ef al., 1955). L'administration de doses excessives d'urée s'accompagne de signes cliniques d'intoxication toujours caractérisés en premier lieu par la paralysie des préestomacs (Lewis, 1960). Celle-ci est complète dès le seuil d'ammoniémie de $4 \mathrm{mg} / \mathrm{l}$ de sang alors que les trémulations musculaires voire les crises tétaniques ef l'hypersalivation sont observables chez le Mouton à partir de 7 à $8 \mathrm{mg} / \mathrm{l}$ (Webb, Barfley et Meyer, 1972).

La nature des troubles métaboliques observés est en partie connue (altération du métabolisme énergétique de la cellule hépatique, inhibition de certaines étapes du cycle de Krebs, formation de phosphates ammoniacomagnésiens et troubles du métabolisme potassique), mais l'existence de chémorécepteurs spécifiques déterminant la sensibilité toute particulière de la motricité rumino-réticulaire reste à démontrer (Wolter, 1974).

Le premier objectif de ce travail a été la détermination du ou des facteurs responsables lors de l'uréolyse de la paralysie rumino-réticulaire : $\mathrm{NH}_{3}$ et/ou élévation du $\mathrm{pH}$. La deuxième partie a été consacrée à la localisation du niveau ou site d'action local, périphérique ou central de l'agent responsable de cette paralysie. 


\section{Matériel et méthodes.}

\section{Préparations chirurgicales.}

Quatre brebis adultes de race Lacaune pesant $45-50 \mathrm{~kg}$, munies de canules permanentes du rumen, ont été placées en cage à métabolisme disposant d'eau et de foin à volonté en dehors des périodes d'expérimentation.

Au préalable, une intervention sous anesthésie générale (au penthobarbital sodique : Nesdonal, N. D.) à la dose de $20 \mathrm{mg} \cdot \mathrm{kg}^{-1}$ a permis la pose d'un fin cathéter (PE 60) dans une branche latérale de la veine ruminale droite ef celle d'un cathéter de large calibre (diam. externe : $2 \mathrm{~mm}$ ) par la veine cæco-colique, jusque dans la veine porte, à $1 \mathrm{~cm}$ en retrait du carrefour des veines hépatiques. Dans un même remps, 2 cathéters ont été placés dans chacune des veines jugulaires à l'aide d'un trocart.

Les contrôles de motricité ont eu lieu entre 14 et $19 \mathrm{~h}$, une heure après le retrait des aliments. Un ballonnet placé dans le sac dorsal du rumen est relié pneumatiquement à un tambour inscripteur de Marey. Le rythme et l'amplitude des contractions du rumen sont mesurés à partir des enregistrements obtenus sur papier noirci.

\section{Protocole expérimental.}

Première série. Une heure après le début des enregistrements des phénomènes moteurs, $250 \mathrm{ml}$ des 3 solutions suivantes ont été introduits au niveau du sac ventral du rumen à l'aide d'un long tuyau de polyvinyl dont les mouvements permettent un brassage grossier du contenu affiné par barbotage d'azote.

- Solution contenant 1,6 mole/l de $\mathrm{NH}_{3}$ à $\mathrm{pH}: 11,1$

- Solution contenant 1,6 mole/l de $\mathrm{NH}_{3}$ à $\mathrm{pH}: 8,8$

- Solution contenant 40,0 mmoles de $\mathrm{NaOH}$ à $\mathrm{pH}: 11,1$.

Des prélèvements de $5 \mathrm{ml}$ de jus de rumen, de sang portal et périphérique ont été effectués à $30 \mathrm{mn}$ d'intervalle en période témoin, puis à 10, 20,30,60 et $90 \mathrm{mn}$ après l'addition ; les contrôles ont été poursuivis par des prélèvements toutes les heures pendant $5 \mathrm{~h}$.

Deuxième série. L'augmentation provoquée de la teneur du sang portal en ammoniaque a été obtenue par la perfusion au niveau de la veine ruminale droite d'une solution contenant 300 mmoles/l de $\mathrm{NH}_{3}$ à raison de $4 \mathrm{ml} . \mathrm{mn}^{-1}$ pendant une heure. Les prélèvements de sang portal, programmés de la même manière que pour la première série d'essais, ont été effectués par le cathéter de gros calibre situé dans la veine porte.

Enfin, la perfusion d'ammoniaque par voie sanguine périphérique a été réalisée au niveau d'une veine jugulaire à partir d'une solution contenant 30 mmoles/l d' $\mathrm{NH}_{3}$ (4 $\mathrm{ml} \cdot \mathrm{mn}^{-1}$ pendant $60 \mathrm{mn}$ ) ; les échantillons de sang sont alors prélevés dans la veine jugulaire contra-latérale.

\section{Dosages.}

La détermination de l'ammoniémie totale du sang et du jus de rumen a été faite sur l'auto-analyseur Technicon selon la méthode décrite par Puył (1973) inspirée 
de la réaction de Berthelot avec mesure préalable de $\mathrm{pH}$ du contenu ruminal dès le prélèvement et l'ajustement thermique.

\section{Résultats.}

\section{Additions intraruminales.}

L'introduction dans le rumen de 400 mmoles de $\mathrm{NH}_{3}$ à $\mathrm{pH}=11,1$ procure après brassage, selon le volume d'ingesta et la vitesse de diffusion de la solution ajoutée, une concentration maximale en $\mathrm{NH}_{3}$ total dans le rumen de 1,15 $\pm 0,22 \mathrm{~g} / \mathrm{I}$ (valeur moyenne voisine de celle observée, $40 \mathrm{mn}$ après addition de $0,5 \mathrm{~g} / \mathrm{kg}$ d'urée, pour un $\mathrm{pH}$ de l'ordre de 8,5) (fig. $1 \mathrm{~A}$ et $\mathrm{B}$ ). La disparition de toute contraction du réticulo-rumen survient en 7 à $8 \mathrm{mn}$; elle dure $19 \pm 6 \mathrm{mn}(\mathrm{n}=12)$ et la levée de cette inhibition laisse subsister des contractions de faible amplitude au-delà de $120 \mathrm{mn}$. La réduction de l'alcalinité des solutions d'ammoniaque infusées par ajustement à $\mathrm{pH}=8,8$ ralentit la vitesse d'absorption à partir du contenu ruminal en déplaçant l'équilibre $\mathrm{NH}_{3} \rightleftharpoons \mathrm{NH}_{4}{ }^{+}$avec un $\mathrm{pH}$ qui n'excède pas 7,1 à l'intérieur du rumen. La durée d'inhibition totale des contractions du réticulo-rumen n'est plus alors que de $13 \pm 4 \mathrm{mn}$ pour une teneur en ammoniac total aussi élevée. $A t=60 \mathrm{mn}$, la fréquence ef l'amplitude des contractions primaires représentent 63 p. 100 et 42 p. 100 des valeurs témoins (53 p. 100 et 31 p. 100 pour $\mathrm{NH}_{3}$ à $\left.\mathrm{pH}=11,1\right)$. L'introduction d'une solution de $\mathrm{NaOH}$ à $\mathrm{pH}=11,1$ provoque une brève élévation du $\mathrm{pH}$ rapidement contrôlée par les systèmes tampons du contenu ruminal (fig. 1 B). La motricité du rumen n'est jamais inhibée, seul un allongement significatif $(P \leqslant 0,05)$ des cycles est de règle 5 à $15 \mathrm{mn}$ après l'administration de la solution (fig. $1 \mathrm{C}$ ). L'occurrence des contractions secondaires du rumen, établie à partir du rapport des nombres de cycles simples et doubles, est réduite significativement $(P \leqslant 0,01)$ de 48 et 37 p. 100 pour les perfusions des solutions ammoniacales à $\mathrm{pH}=11,1 \epsilon+8,8$.

\section{Perfusion par voie sanguine portale et périphérique.}

En perfusant par la veine ruminale droite une solution contenant 300 mmoles/l $\mathrm{d}^{\prime} \mathrm{NH}_{3}$, il est possible d'obtenir des concentrations sanguines dans la veine porte voisines de $2,5 \mathrm{mg}$ p. 100 et équivalentes à celles obtenues avec une addition dans le rumen de 400 mmoles d' $\mathrm{NH}_{3}$ à $\mathrm{pH}=8,8$ (fig. $2 \mathrm{~A}$ ).

Pendant toute la durée de perfusion, la fréquence des contractions du rumen ne diffère pas de la valeur de départ et seule l'ampliłude a été affectée significativement $(P \leqslant 0,05)$ pour 2 sujets; ces dernières variations n'apparaissent pas significatives sur l'ensemble des 8 essais pour les 4 brebis (fig. 2 B).

En effectuant au niveau de la jugulaire la perfusion d'une solution d'ammoniac moins concentrée que celle introduite dans la veine porte $(30 \mathrm{mmcles} / \mathrm{l})$, mais pour laquelle l'ammoniémie (périphérique) est supérieure à celle observée par addition d' $\mathrm{NH}_{3}$ dans le rumen, aucun effet moteur immédiat $(10-30 \mathrm{mn})$ n'est retrouvé alors que les concentrations dans le sang veineux prélevé à la veine jugulaire contralatérale peuvent atteindre $0,7 \mathrm{mg}$ p. 100 (fig. 2 B). 

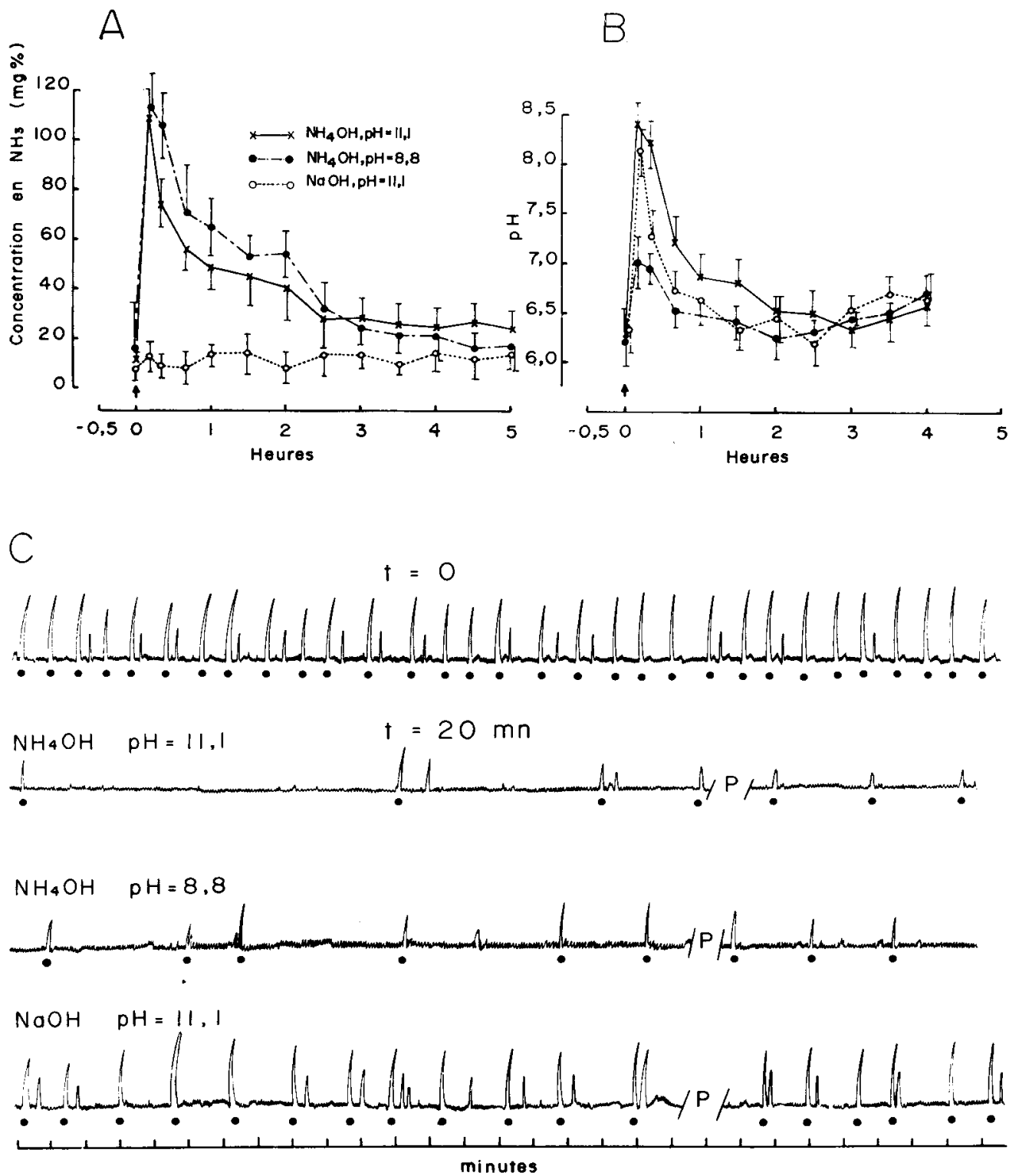

FIG. 1. - Influence de la teneur en ammoniaque et du pH sur la motricité du rumen chez la brebis.

En $A$ et $B$, les flèches verticales indiquent le moment de l'addition dans le rumen de $250 \mathrm{ml}$ de solutions contenant 400 mmoles d' $\mathrm{NH}_{3}(\mathrm{pH}=11,1$ et 8,8$)$ ou 40 mmoles de $\mathrm{NaOH}(\mathrm{pH}=11,1)$.

En $\mathrm{C}$, mécanogrammes des contractions du rumen, obtenus par la mesure des pressions au niveau du sac dorsal.

Pour les 2 valeurs de $\mathrm{pH}$, la présence d'ammoniaque détermine une inhibition en fréquence et en amplitude des contractions du rumen, qui n'est pas observée dans l'alcalinisation par addition de $\mathrm{NaOH}$. 
Une diminution d'environ 20 p. 100 de l'amplitude des contractions primaires du rumen esł de règle en fin de perfusion ; la fréquence d'apparition des contractions secondaires reste inchangée pour les 2 types de perfusion envisagés.
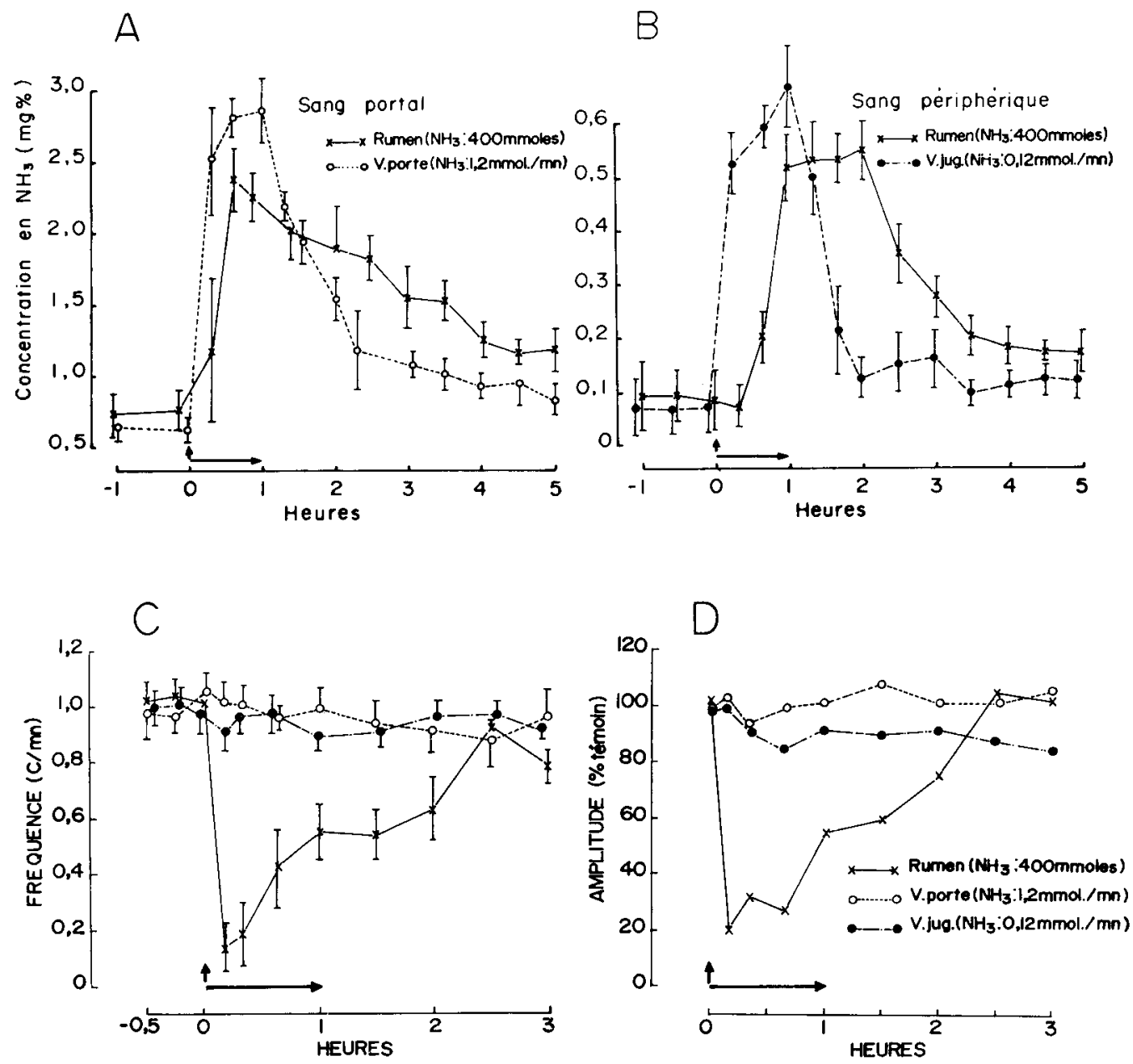

FIG. 2. - Modifications de l'ammoniémie locale ou périphérique et de la motricité du rumen provoquées par un apport sanguin ou ruminal d'ammoniaque.

En A : Elévations de l'ammoniémie portale, obtenues par infusion ( $\uparrow$ ) de 400 mmoles d' $\mathrm{NH}_{3}$ dans le rumen $(x-x)$ ou par une perfusion prolongée $(\rightarrow)$ d'une solution d'ammoniaque dans la

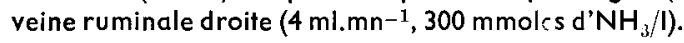

En B : Contre-épreuve d'augmentation de l'ammoniémie périphérique par perfusion d'une heure d'une solution d'ammoniaque dans la veins iugulaire.

En $C$ et $D$ : Effets comparés de ces perfusions a'ammoniaque sur la fréquence (C) ef l'amplitude (D) des contractions primaires du rumen. 


\section{Discussion.}

Le déséquilibre entre la vitesse de production d'ammoniaque dans l'uréolyse ef celle de son absorption par la paroi du rumen est responsable de son accumulation à ce niveau.

L'absorption intra-ruminale se fait sous la forme libre $\mathrm{NH}_{3}$; elle est donc favorisée par une élévation de $\mathrm{pH}$ (Lewis, Hill et Annison, 1957) ; ainsi, une augmentation de 1 unité $\mathrm{pH}$ entre 7,2 et 8,2 multiplie par 10 la concentration en ammoniaque diffusible $\left(\mathrm{NH}_{3}\right)$ et par conséquent son absorption (Hemingway, Parkins et Riłchie, 1972). Le seuil d'intoxication hépatique ou métabolique est déterminé par la valeur du rapport $\mathrm{NH}_{3} / \mathrm{NH}_{4}{ }^{+}$.

Par contre, la motricité du rumen, bien qu'alcalino-dépendante, est également sensible aux formes $\mathrm{NH}_{3}$ et $\mathrm{NH}_{4}{ }^{+}$. En effet, la durée de l'inhibition motrice est seulement réduite de $35 \mathrm{p}$. 100 alors que le $\mathrm{pH}$ dans le rumen, $10 \mathrm{mn}$ après addition d'ammoniaque, passe de 6,2 à 8,4 correspondant à des variations allant de 1 à 100 de la concentration en $\mathrm{NH}_{3}$ libre.

Un tel résultat suggère une chémosensibilité locale puisque seule la forme $\mathrm{NH}_{3}$ est absorbée et peut être véhiculée jusqu'au foie. L'action locale est démontrée par l'absence de réponses motrices à des perfusions veineuses portale ou périphérique.

Quant aux symptômes d'intoxication aiguë, ils ne sont en fait perceptibles que pour une saturation hépatique telle que l'on retrouve une concentration veineuse périphérique en $\mathrm{NH}_{3}$ de l'ordre de 0,4 à $0,5 \mathrm{mg} \mathrm{p}$. 100 (Webb, Bartley et Meyer, 1972). Ainsi, la neutralisation de l'alcalinité du contenu du rumen par l'addition d'acides relève le seuil d'intoxication et réduit l'anorexie consécutive à une surcharge ammoniacale (observations personnelles), mais elle laisse persister une forte parésie du rumen.

En définitive, l'excès d'ammoniaque est responsable de la paralysie des préestomacs dans le cas d'intoxication par une surcharge en azote non protéique dans l'alimentation. Cette chémosensibilité est locale et tout traitement préventif ou thérapeutique contre l'alcalose par voie sanguine ne permet pas une régression immédiate des troubles moteurs.

Commission CNERNA Digestion-Absorption, Tours, 13 novembre 1976.

\section{Références}

HEMINGWAY R. G., PARKINS J. J., RITCHIE N. S., 1972. Comparative effects of oral administration of urea and urea phosphate on the concentration of ammonia and urea in the blood of sheep. Brit. vef. J., 128, 82-92.

LEWIS D., 1960. Ammonia toxicity in the ruminant. J. agric. Sci., 55, 187-197.

LEWIS D., HILL K. J., ANNISON E. F., 1957. Studies on the portal blood of sheep. I. Absorption of ammonia from rumen of the sheep. Biochem. J., 66, 587-672.

PUYT J. D., 1973. Contribution à l'étude de l'ammoniémie du chien. Thèse Doct. Vet., Toulouse, 102 Pp.

REPP W.W., HALE W. H., CHENG E. W., BURROUGHS W., 1955. The influence of oral administration of non protein nitrogen feeding compounds upon blood ammonia and urea levels in lambs. J. anim. Sci., 14, 118-124.

WEBB D. W., BARTLEY E. E., MEYER R. M., 1972. A comparison of nitrogen metabolism and ammonia toxicity from ammonium acetate and urea in cattle. J. anim. Sci., 35, 1263-1270.

WOLTER R., 1974. L'azote non proféique dans l'alimentation des ruminants. I. Mode de valorisation et tolérance à l'urée, Rev. Med. vét., 125, 761-779. 